\title{
Studies on Structural, Spectral, and Optical Properties of Organic Nonlinear Optical Single Crystal: 2-Amino-4,6-dimethylpyrimidinium p-Hydroxybenzoate
}

\author{
S. Sudhahar, ${ }^{1}$ M. Krishna Kumar, ${ }^{1}$ A. Silambarasan, ${ }^{1}$ \\ R. Muralidharan, ${ }^{2}$ and R. Mohan Kumar ${ }^{1}$ \\ ${ }^{1}$ Department of Physics, Presidency College, Chennai 600 005, India \\ ${ }^{2}$ Department of Science and Humanities, Veltech High Tech Dr. R. Rangarajan Dr. Sakunthala Engineering College, \\ Chennai 600 062, India \\ Correspondence should be addressed to R. Mohan Kumar; mohan66@hotmail.com
}

Received 31 December 2012; Accepted 7 March 2013

Academic Editor: Matjaz Valant

Copyright (c) 2013 S. Sudhahar et al. This is an open access article distributed under the Creative Commons Attribution License, which permits unrestricted use, distribution, and reproduction in any medium, provided the original work is properly cited.

\begin{abstract}
2-Amino-4,6-dimethylpyrimidinium p-hydroxybenzoate (ADPHB), a novel organic material, was synthesized, and crystals were grown by slow evaporation solution growth method. The cell parameters and crystalline perfection of the grown crystal were studied by single crystal and powder X-ray diffraction analyses. The presence of various functional groups of the grown crystal was confirmed using Fourier Transform infrared spectral analysis. UV-Visible spectrum shows that ADPHB crystals have high transmittance in the range 305-900 $\mathrm{nm}$. The refractive index and transient photoluminescence properties of the grown crystal were analyzed. The laser induced surface damage threshold, optical birefringence, and second harmonic generation efficiency of the grown crystal were studied.
\end{abstract}

\section{Introduction}

The research on new organic nonlinear optical (NLO) materials has attracted more research groups to them for their advantages over the inorganic nonlinear optical materials. The organic nonlinear optical molecules generally have a larger second-order nonlinear optical coefficient, and hence they are widely used in many applications such as optical communication, information storage, and optical switching [1-5]. The continuous research on organic NLO materials prompted to find many new nonlinear optical complexes engineered by organic molecules. For instance, nonlinear optical complexes formed from pyridine and carboxylic acids have been crystallized, and the structural, optical, and thermal properties of these crystals have been investigated due to their significant impact on laser technology, optical communication, and optical data storage technology [6]. Organic nonlinear optical compounds have large macroscopic second-order NLO susceptibilities $\chi^{(2)}$, low dielectric dispersion, and ultrafast response to external electric fields $[7,8]$. Organic nonlinear optical materials are formed based on NLO chromophores, which should be oriented in noncentrosymmetric way in order to exhibit macroscopic secondorder nonlinear optical response. There are several different strategies resulting in macroscopic $\chi^{(2)}$ organic materials, including ferroelectric liquid crystals [9], self-assembled films [10], Langmuir-Blodgett films [11], and poled polymer films [12], as well as single crystals [13]. Among them, polar organic crystals are of special interest, since large NLO effect can be achieved due to the high density of chromophores in the crystals, as well as the most stable packing of chromophores in macroscopic materials, resulting in a superior photochemical and thermal stability as compared with soft category materials [14]. Furthermore, organic NLO crystals can be produced in a bulk form and are therefore not limited to thin-film applications [13-16]. This is particularly important for frequency conversion applications such as terahertz-wave generation, where large interaction lengths 

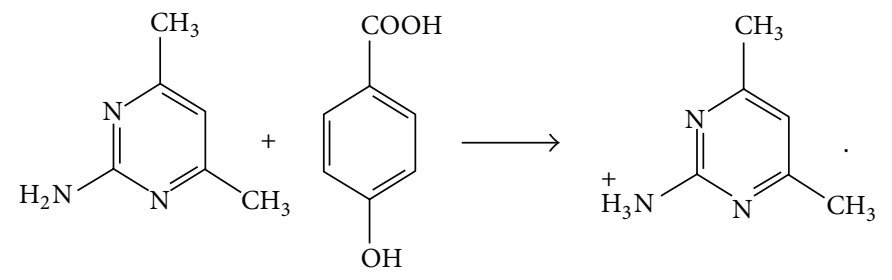<smiles>O=C([O-])c1ccc(O)cc1</smiles>

FIGURE 1: Material synthesis scheme of ADPHB.

are required [17]. The asymmetric unit of 2-amino-4,6dimethylpyrimidinium p-hydroxybenzoate (ADPHB) contains a 2-amino-4,6-dimethylpyrimidine (AMPY) molecule and a 4-hydroxybenzoic acid (4-HBZ) molecule. Both species are neutral; thus ADPHB is an adduct rather than a molecular salt. The atoms $\mathrm{O}_{2}$ and $-\mathrm{N}_{2} \mathrm{H}_{2}$ group act as hydrogenbond donors to atoms $\mathrm{N}_{1}$ and $\mathrm{O}_{3}$, respectively [18]. In the present investigation, single crystal of the title compound has been grown by slow evaporation method. The grown crystals were subjected to single crystal and powder X-ray diffraction, FTIR, thermal, UV-Visible transmission, refractive index, photoluminescence (PL), laser induced surface damage threshold, birefringence, and second harmonic generation studies.

\section{Experimental}

2.1. Material Synthesis and Crystal Growth. Commercially available starting materials, 2-amino-4,6-dimethylpyrimidine $\left(\mathrm{C}_{6} \mathrm{H}_{9} \mathrm{~N}_{3}\right)$ (Sigma Aldrich, 99\%) and p-hydroxybenzoic acid $\left(\mathrm{C}_{7} \mathrm{H}_{6} \mathrm{O}_{3}\right)$ (Loba Chemie, 99\%), were dissolved in methanol. The clear ADPHB solution was obtained after a continuous stirring for about six hours to achieve homogeneous mixture of the solution. Then, the growth solution was allowed for slow evaporation which yielded the spontaneously nucleated crystals of the synthesized compound. The synthesized compound was further purified by successive recrystallization process in methanol, and it was utilized for the crystal growth. The chemical synthesis scheme of the ADPHB compound is shown in Figure 1.

Single crystal of the ADPHB was grown by slow evaporation method in methanol solution. The saturated solution was prepared by dissolving the purified ADPHB salt in methanol using solubility data. It was stirred well for about $6 \mathrm{~h}$ to obtain a homogeneous mixture of the growth solution and kept in the constant temperature bath at $38^{\circ} \mathrm{C}$ with an accuracy of $\pm 0.01^{\circ} \mathrm{C}$. The well-defined ADPHB single crystals with good transparency were collected within the period of 15-18 days with an average size of $5 \mathrm{~mm} \times 5 \mathrm{~mm} \times 1.5 \mathrm{~mm}$ as shown in Figure 2.

2.2. Characterization Techniques. The grown single crystal of ADPHB was characterized by different instrumental methods. Single crystal and powder X-ray diffraction analyses were carried out to estimate the lattice parameters and crystalline quality of the grown crystal using Bruker Nonius CAD-4/MACH 3 single X-ray diffractometer and Bruker
AXS CAD4 diffractometer, respectively. FT-IR spectrum was recorded for ADPHB using JASCO FTIR 410 spectrometer, and it was effectively used to identify the functional groups of the synthesized compound. TG-DTA analyses were carried out using SII TG/DTA 6300 EXSTAR apparatus with a heating rate of $10^{\circ} \mathrm{C} / \mathrm{min}$ under $\mathrm{N}_{2}$ atmosphere. The optical transmission spectrum of ADPHB crystal was recorded with PerkinElmer Lambda 35 Spectrophotometer in the range of $190-1100 \mathrm{~nm}$. The photoluminescence property of grown crystal was studied using RF-5301 Spectrometer. The laser damage threshold was recorded for ADPHB using $1064 \mathrm{~nm}$ wavelength of $\mathrm{Nd}$ :YAG laser radiation. The optical birefringence was observed using modified channeled spectrum method, and the relative second harmonic generation efficiency was evaluated by Kurtz-Perry powder technique using $\mathrm{Nd}$ : YAG laser source.

\section{Results and Discussion}

3.1. X-Ray Diffraction Analyses. The cell parameters of ADPHB crystal were obtained from single crystal XRD data collected at room temperature. The ADPHB compound crystallizes in monoclinic crystal system with noncentrosymmetric space group Cc. The estimated cell parameters are $a=9.025 \AA, b=11.469 \AA, c=12.580 \AA, \alpha=\gamma=90^{\circ}, \beta=$ $102.804^{\circ}$, and $v=1270 \AA^{3}$ and are in good agreement with the reported data [18]. The ADPHB crystal was crushed to a uniform fine powder and was subjected to powder $\mathrm{X}$ ray diffraction study to reveal crystalline perfection of the compound. The observed sharp and intense peaks reveal the high crystallinity of the grown crystal and the corresponding peaks were indexed. The recorded powder X-ray diffraction pattern of ADPHB crystal is shown in Figure 3.

3.2. FT-IR Spectral Analysis. The FTIR spectrum was recorded to understand the chemical bonding, and it provides useful information regarding the molecular structure of the compound. FTIR spectrum was taken for the powdered sample in the wavelength range $4000-400 \mathrm{~cm}^{-1}$ as shown in Figure 4. The $\mathrm{KBr}$ pellet technique was used to analyze the sample. The powdered ADPHB sample with $\mathrm{KBr}$ in the ratio of 1:100 was used to make the pellet. The peak at $3476 \mathrm{~cm}^{-1}$ is due to phenolic $\mathrm{O}-\mathrm{H}$ stretching. The peak at 3292 is due to $\mathrm{NH}$ stretching vibration of primary amine group in 2-amino-4,6-dimethylpyrimidine. The absorption at $3065 \mathrm{~cm}^{-1}$ is due to $\mathrm{C}-\mathrm{H}$ stretching. The peaks at 2969 and 


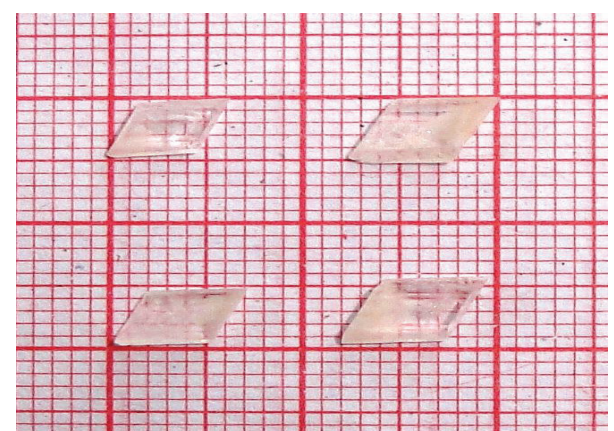

FIGURE 2: Photograph of as-grown ADPHB crystals.

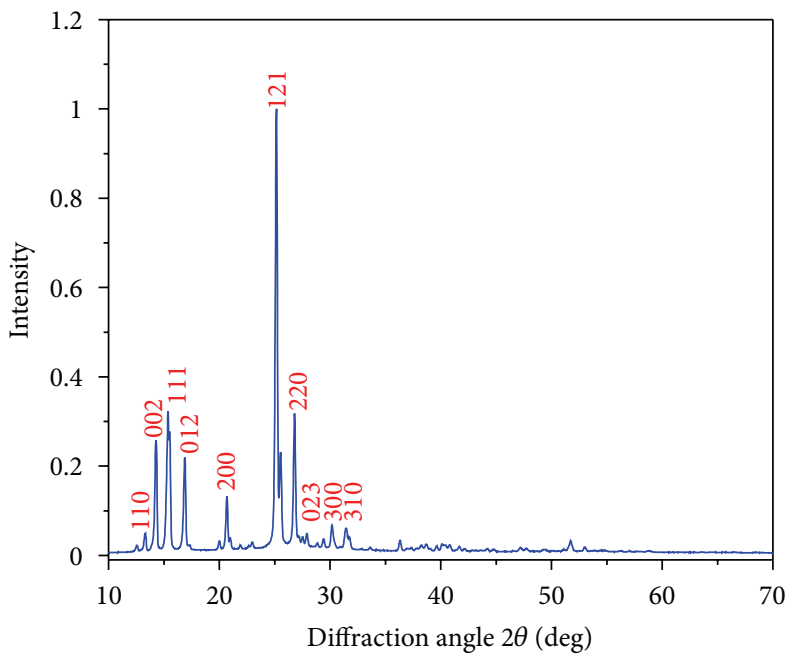

FIGURE 3: Powder XRD pattern of ADPHB crystal.

$2926 \mathrm{~cm}^{-1}$ are due to $\mathrm{O}-\mathrm{H}$ stretch of carboxylic acid group present in the compound. The characteristic $\mathrm{C}=\mathrm{O}$ carbonyl stretching vibration appears as a sharp peak at $1681 \mathrm{~cm}^{-1}$. The $\mathrm{C}=\mathrm{N}$ stretching vibration appears at $1628 \mathrm{~cm}^{-1}$. The $\mathrm{N}-\mathrm{H}$ bend primary amine produces absorption peak at $1595 \mathrm{~cm}^{-1}$. The absorption peaks at $1366 \mathrm{~cm}^{-1}$ are due to C$\mathrm{N}$ stretch in aromatic amines. The aromatic $\mathrm{C}-\mathrm{H}$ in-plane bending vibration appears at 853,830 , and $777 \mathrm{~cm}^{-1}$. The assigned vibrational frequencies of functional groups of title compound are listed in Table 1.

3.3. Thermal Analysis. The thermal stability of powdered ADPHB sample was studied by thermogravimetric analysis (TGA) and differential thermal analysis (DTA) in the nitrogen atmosphere with a heating rate of $10^{\circ} \mathrm{C} / \mathrm{min}$. The ADPHB sample weighing $1.577 \mathrm{mg}$ was used to record TGA and DTA thermogram which is shown in Figure 5. In the TGA curve, there is no weight loss peak that occurs before $130.4^{\circ} \mathrm{C}$ and it reveals that there is no lattice water molecule in the compound. TG curve shows single stage weight loss patterns it is observed from the temperature $130.4^{\circ} \mathrm{C}$ up to the $231.9^{\circ} \mathrm{C}$ and it is approximately $97.1 \%$ percentage loss of the compound. Thus, the TGA curve indicates that the sample is stable up to the temperature of $130.4^{\circ} \mathrm{C}$ for nonlinear optical

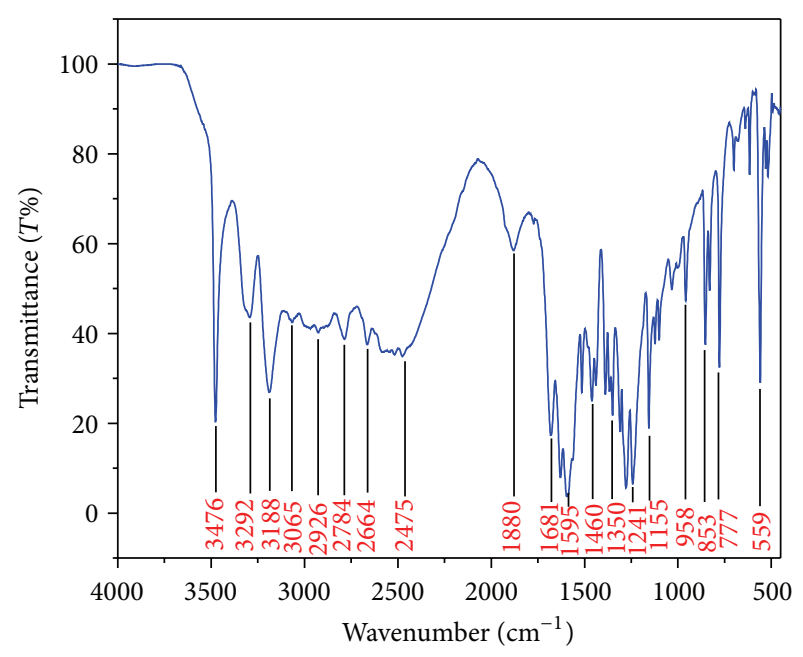

FIgURE 4: FTIR spectrum of ADPHB.

TABLE 1: FTIR frequency assignments of ADPHB.

\begin{tabular}{lc}
\hline Functional group assignments & ADPHB sample \\
\hline O-H phenolic stretching & 3476 \\
N-H primary amines & 3292 \\
C-H stretching & 3065 \\
O-H carboxylic stretching & 2969 and 2926 \\
C=N stretching & 1628 \\
C=O carbonyl stretching & 1681 \\
N-H bend primary amines & 1595 \\
C-N stretch in aromatic amines & 1366 \\
C-H bending & 853,830, and 777 \\
\hline
\end{tabular}

applications. In the DTA curve, there are two endothermic steps available. The observed first endothermic peak at $183.4^{\circ} \mathrm{C}$ and a second endothermic peak at $219^{\circ} \mathrm{C}$ are due to the removal of the hydroxyl and amino groups expelled from the compound respectively $[19,20]$. Hence, the recorded thermogram of the compound confirms ADPHB compound, and it is confirmed that the compound is thermally stable up to $130.4^{\circ} \mathrm{C}$.

\subsection{Optical Studies}

3.4.1. UV-Visible Transmission Studies. UV-Visible spectral analysis gives useful information about electronic transitions in the compound [21]. The optical behavior of ADPHB crystal specimen was analyzed using UV-Vis spectrophotometer in the wavelength range of $190-900 \mathrm{~nm}$. For this study, an optically polished single crystal of thickness $1.5 \mathrm{~mm}$ was used and the recorded transmittance spectrum is shown in Figure 6. The cut-off wavelength of the crystal is observed at $305 \mathrm{~nm}$, and the crystals show good transmittance percentage in the range of $330-900 \mathrm{~nm}$. This spectral study would be assisted in the understanding of electronic structure and the optical band gap of the crystal. From the spectrum, it is observed that ADPHB crystal has high transmittance in the 


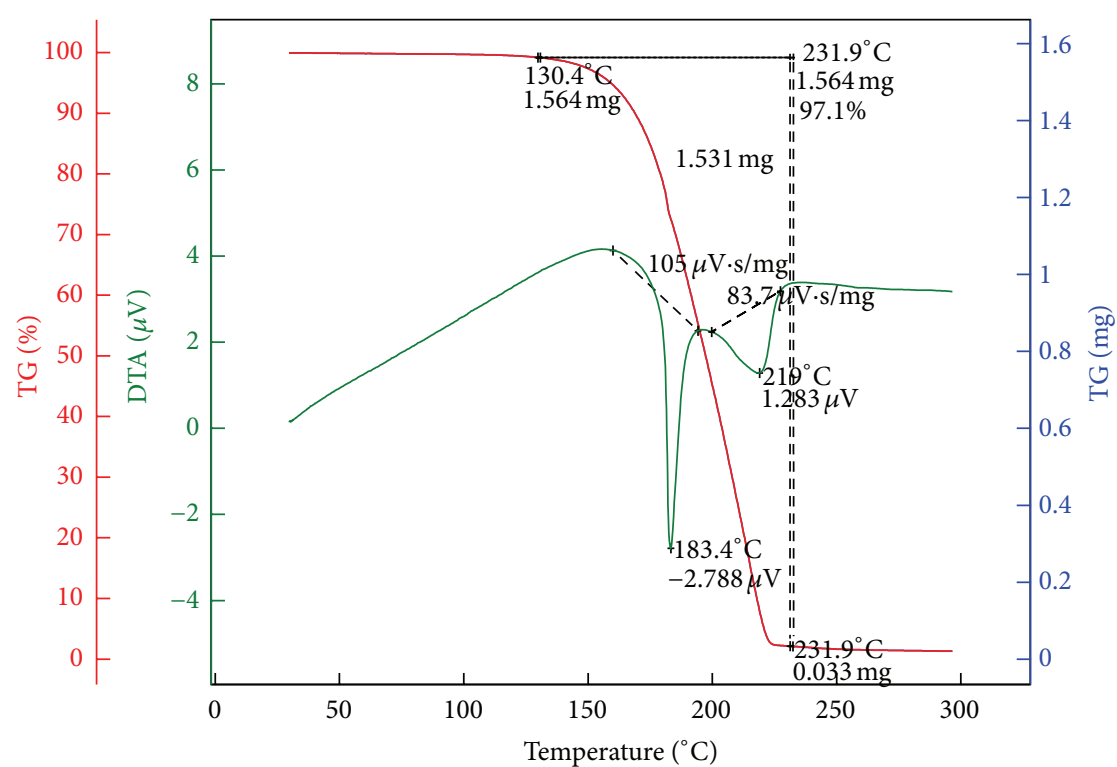

Figure 5: TG/DTA trace of ADPHB.

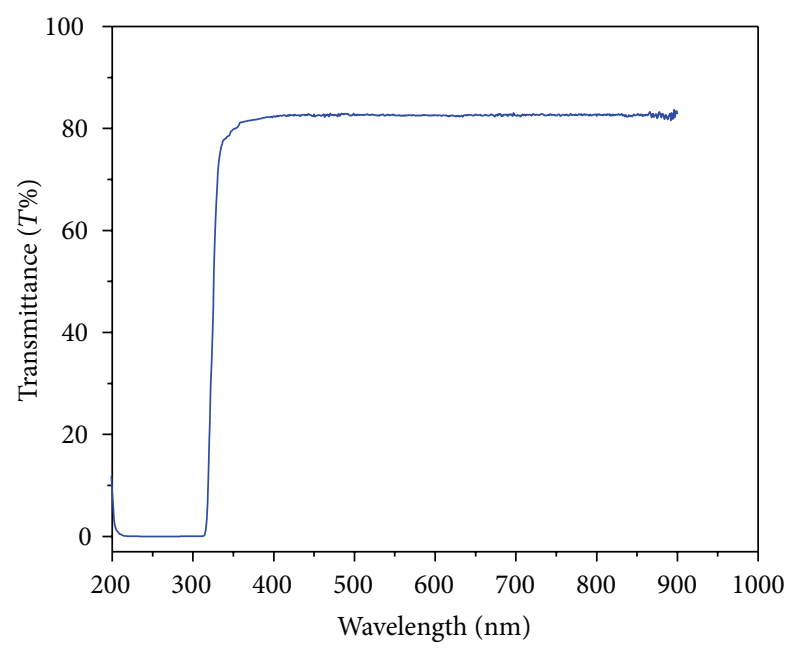

Figure 6: UV-visible transmittance spectrum of ADPHB single crystal.

entire visible, near infrared region and this property enables the material holds good for optoelectronic applications.

3.4.2. Optical Band Gap Energy $\left(E_{g}\right)$ Calculation. For optical device fabrication, the crystal should have high transparency in a considerable range of wavelength [22]. The UV cut-off wavelength of the grown ADPHB crystal was found to be $305 \mathrm{~nm}$, and it is useful to make them potential material for optical device fabrication. The optical absorption coefficient $(\alpha)$ was calculated using the relation,

$$
\alpha=\left(\frac{1}{d}\right) \log \left(\frac{1}{T}\right)
$$

where $d$ is the thickness of the crystal and $T$ is the transmittance. Owing to the direct band gap, the crystal under

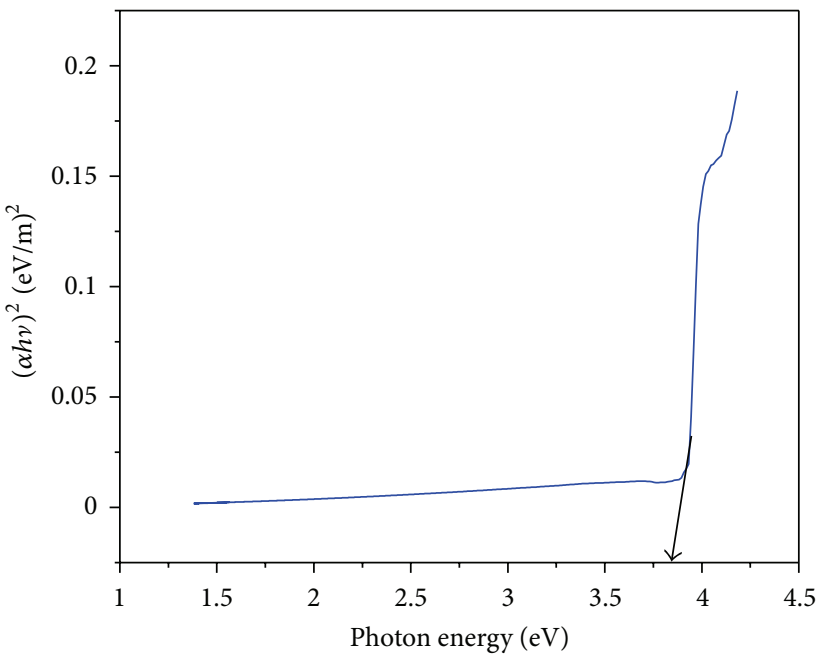

FIGURE 7: Plot of photon energy versus $(\alpha h \nu)^{2}$ of ADPHB crystal.

study has an absorption coefficient $(\alpha)$ obeying the following relation for high photon energies $(h \nu)$,

$$
h \nu \alpha=A\left(h \nu-E_{g}\right)^{1 / 2}
$$

where $A$ is a constant, $E_{g}$ is the optical band gap, $h$ is the Planck's constant, and $v$ is the frequency of the incident photons [23]. The band gap of grown ADPHB crystal was estimated by plotting $(\alpha h \nu)^{2}$ versus $h v$ and it is shown in Figure 7. The band gap energy of grown ADPHB crystal was found to be $3.84 \mathrm{eV}$. This indicates the consequences of wide band gap and large transmittance of the grown crystal.

3.4.3. Determination of Optical Constants. The optical behaviour of materials is important to determine their usage in 
optoelectronic devices [24]. The study of optical constants of a material such as refractive index and extinction coefficient is quite essential to examine the material's potential optoelectronic applications [25]. Further, the optical properties may also be closely related to the material's atomic structure, electronic band structure and electrical properties. The optical constants $(n, k)$ were determined from the transmission $(T)$ and reflection $(R)$ spectrum based on the following relations [26]:

$$
T=\frac{(1-R)^{2} \exp (-\alpha t)}{1-R^{2} \exp (-2 \alpha t)},
$$

where $t$ is the thickness and $\alpha$ is related to extinction coefficient.

$k$ value was determined by

$$
k=\frac{\alpha \lambda}{4 \pi} .
$$

The refractive index $(n)$ can be determined from the reflectance $(R)[27]$

$$
R=\frac{(n-1)^{2}+k^{2}}{(n+1)^{2}+k^{2}}
$$

The reflectance in terms of absorption coefficient was determined using

$$
R=1 \pm \frac{\sqrt{1-\exp (-\alpha t)+\exp (\alpha t)}}{1+\exp (-\alpha \mathrm{t})}
$$

and from the above equation, the refractive index $n$ can be derived as

$$
n=\frac{-(R+1) \pm \sqrt{-3 R^{2}+10 R-3}}{2(R-1)} .
$$

The calculated refractive index value using the above equations for the grown ADPHB crystal is 2.562. The other calculated values of extinction coefficient $(k)$ and reflectance $(R)$ are 0.00310 and 0.49 , respectively.

3.4.4. Photoluminescence (PL) Spectral Studies. Photoluminescence spectroscopy is a nondestructive method of probing the electronic structure of materials. The photoluminescence spectrum was recorded for the as-grown inclusion free single crystal of $\mathrm{ADPHB}$ at room temperature with an excitation wavelength of $316 \mathrm{~nm}$ as shown in Figure 8. A very strong intense emission peak was observed at $354 \mathrm{~nm}(3.502 \mathrm{eV})$, which corresponds to near band-edge excitons of as-grown crystals. Moreover, absence of visible emission bands has indicated the high crystal quality and perfect crystallinity of as-grown crystals. Therefore, the as-grown ADPHB crystals might be suitable for UV filters and optoelectronic laser devices.

3.4.5. Surface Laser Damage Threshold (LDT) Measurement. One of the essential criteria for the NLO crystal to perform as a device is that it needs high laser damage resistance because

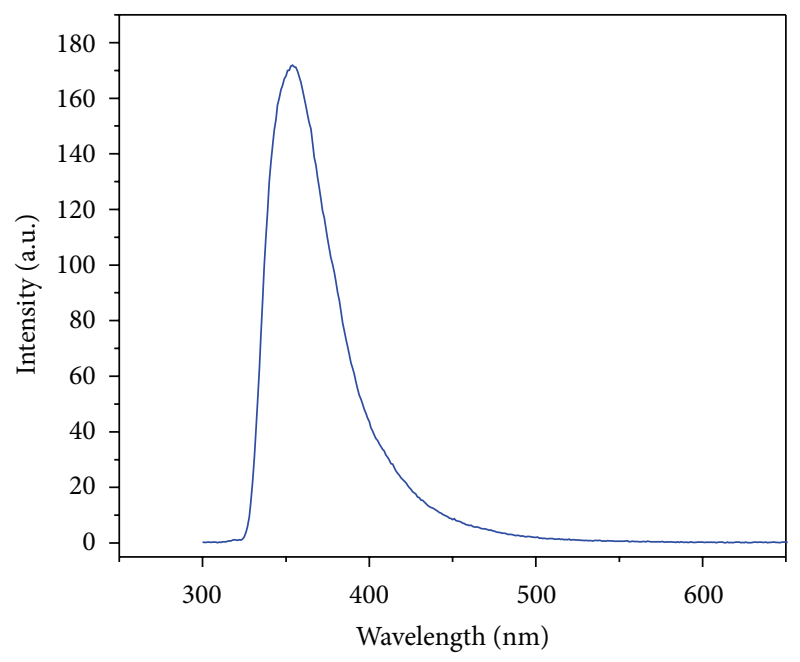

FIgURE 8: Photoluminescence spectrum of ADPHB crystal.

TABLE 2: Comparison of laser damage threshold value of ADPHB crystal with other crystals.

\begin{tabular}{lc}
\hline Crystal & Laser damage threshold $\left(\mathrm{GW} / \mathrm{cm}^{2}\right)$ \\
\hline KDP & 0.2 \\
Urea & 1.5 \\
Beta-barium borate & 5.0 \\
L-arginine phosphate & 10.0 \\
ADPHB & 4.73 \\
\hline
\end{tabular}

high optical intensities are involved in nonlinear process [28]. In the present experiment, an actively Q-switched Nd:YAG laser source with $6 \mathrm{~ns}$ pulse width and $10 \mathrm{~Hz}$ repetition rate was used. The output intensity of the laser was controlled with variable attenuator and delivered to the test sample located at the near focusing of the converging lens. The energy density of the input laser beam was recorded using power meter till the crystal got damaged. The surface damage threshold of the crystal was calculated using the formula

$$
\text { Power density } P_{(d)}=\frac{E}{\tau A} \text {, }
$$

where $E$ is the energy (mJ), $\tau$ is the pulse width (ns) and $A$ is the area of the circular spot size. Multiple shot LDT measurements were made on the well-polished plane of the grown ADPHB crystal. The beam spot size on the sample was $1 \mathrm{~mm}$, and input energy was measured as $40 \mathrm{~mJ}$. The calculated laser damage threshold is $4.73 \mathrm{GW} / \mathrm{cm}^{2}$. The laser damage property is nearly 23.65 times higher than other standard NLO materials of potassium dihydrogen phosphate (KDP) $\left(0.20 \mathrm{GW} / \mathrm{cm}^{2}\right)$ and 3.15 times higher than that of urea $\left(1.5 \mathrm{GW} / \mathrm{cm}^{2}\right)$. The laser damage threshold value of the present sample is compared with other NLO crystals [29] and is given in Table 2. The higher value of LDT will be useful for making laser based devices.

3.4.6. Birefringence Studies. The experimental setup of modified channel spectrum (MCS) method was employed to reveal 


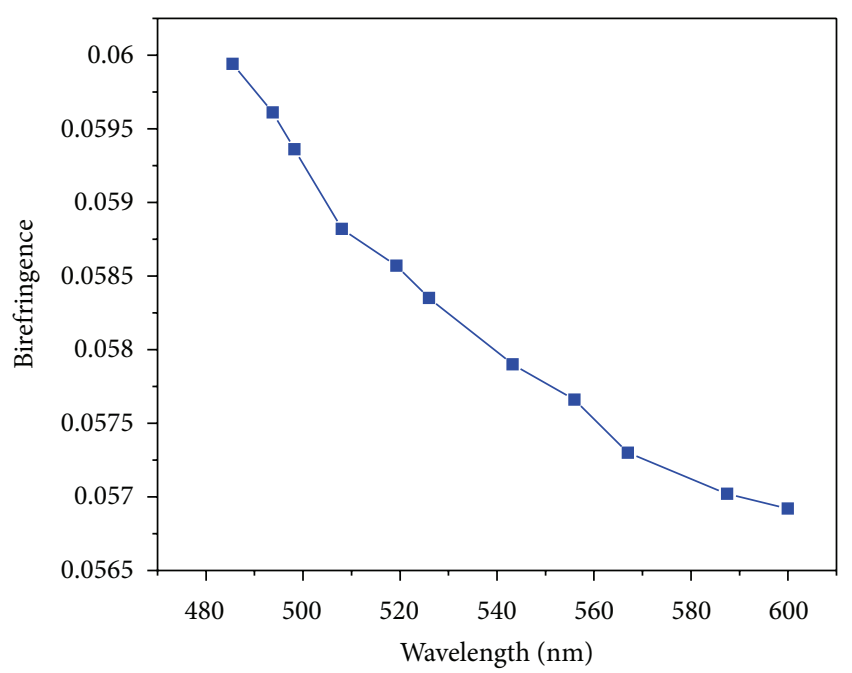

FIgURE 9: Birefringence spectrum of ADPHB crystal.

the birefringence behavior of the specimen $[30,31]$. The constant deviation spectrometer was first calibrated using the standard procedure and $1000 \mathrm{~W}$ tungsten halogen lamp was used as source, which was collimated using a collimator. The optically polished ADPHB single crystal was placed on a rotary stage between crossed polarizer in the path of polarized light. The thickness of about $0.85 \mathrm{~mm}$ ADPHB crystal was used for the measurement. The direction of optical axis of the crystal slab was kept perpendicular to the direction of the collimated light transmitted by the polarizer. Finally, the light was passed through the analyzer and emerging light waves were examined by the microscope. The birefringence was calculated using the relation [32]

$$
\Delta n=\frac{k \lambda}{t}
$$

where $\lambda$ is the wavelength of light, $t$ is the thickness of the crystal and $k$ is the order of interference maximum. The plot drawn between birefringence $(\Delta n)$ and wavelength $(\lambda)$ is shown in Figure 9. From this measurement, the dispersion of birefringence existing in the entire visible region observed.

3.4.7. Second Harmonic Generation Test. The second harmonic generation efficiency of ADPHB crystal was measured by using Kurtz-Perry powder SHG technique with potassium dihydrogen phosphate (KDP) crystal as reference material [33]. The fundamental laser beam of $1064 \mathrm{~nm}$ wavelength and $8 \mathrm{~ns}$ pulse width, with $10 \mathrm{~Hz}$ pulse rate, was made to fall normally on the sample cell. The input laser energy incident on the powdered sample was chosen to be $5.65 \mathrm{~mJ} / \mathrm{pulse}$. The grown single crystal of ADPHB and reference KDP was ground in a uniform particle size of $125-150 \mu \mathrm{m}$, packed in a microcapillary of uniform bore size, and exposed to laser radiations. The fundamental beam was filtered by using an IR filter. A photomultiplier tube was used as detector. The output from the sample was monochromated to collect the intensity of $532 \mathrm{~nm}$ component and to eliminate the fundamental radiation. The generation of the second harmonic was confirmed by the emission of green light. The SHG output efficiency for ADPHB and KDP samples was found to be $195 \mathrm{mV}$ and $55 \mathrm{mV}$, respectively. Thus, it is observed that SHG efficiencies of ADPHB crystal were found to be 3.5 times higher those that of KDP.

\section{Conclusion}

Nonlinear optical 2-amino-4,6-dimethylpyrimidine p-hydroxybenzoate single crystals were grown by slow evaporation method. The crystalline perfection of the grown crystal was examined by X-ray diffraction studies. The modes of vibration molecule groups present in the grown crystal were confirmed by Fourier transform infrared spectral analysis. The thermogravimetric analyses reveal that the crystal is thermally stable up to $130.4^{\circ} \mathrm{C}$ without any structural phase transition. The transmission percentage, lower cutoff wavelength, and optical band gap energy $\left(E_{g}\right)$ of the grown crystal were examined by UV-Vis spectral analysis. The linear refractive index, photoluminescence behavior, and birefringence property of the crystal were examined. The surface laser damage threshold value for ADPHB crystal was found to be $4.73 \mathrm{GW} / \mathrm{cm}^{2}$ and compared with other NLO crystals. The second harmonic generation efficiency of the crystal was found to be 3.5 times higher than that of standard KDP crystal.

\section{Acknowledgment}

One of the authors (M. K. Kumar) would like to acknowledge, Council of Scientific and Industrial Research, New Delhi, India, for providing financial support (project no. 03 (1200)/11/EMR-II).

\section{References}

[1] T. Kaino, B. Cai, and K. Takayama, "Fabrication of DAST channel optical waveguides," Advanced Functional Materials, vol. 12, no. 9, pp. 599-603, 2002.

[2] P. N. Prasad and D. J. Williams, Introduction to Nonlinear Optical Effects in Molecules and Polymers, John Wiley \& Sons, New York, NY, USA, 1991.

[3] B. K. Periyasamy, R. S. Jebas, and B. Thailampillai, "Synthesis and spectral studies of 2-aminopyridiniumpara-nitrobenzoate: a novel optoelectronic crystal," Materials Letters, vol. 61, no. 7, pp. 1489-1491, 2007.

[4] R. W. Munn and C. N. Ironside, Principles and Applications of Nonlinear Optical Materials, Chapman and Hall, London, UK, 1993.

[5] J. Bardan, R. Hierle, A. Perigaud, J. Zyss, and D. J. Williams, Nonlinear Optical Properties of Organic Molecules and Polymeric Materials, vol. 223 of ACS Series, American Chemical Society, Washington, 1993.

[6] M. L. Caroline and S. Vasudevan, "Growth and characterization of an organic nonlinear optical material: 1-alanine alaninium nitrate," Materials Letters, vol. 62, pp. 2245-2248, 2008.

[7] B. J. Coe J.A. Harris, A. K. Clays, G. Olbrechts, A. Persoons, and J. T. Hupp, "Quadratic nonlinear optical properties of $N$ aryl stilbazolium dyes," Advanced Functional Materials, vol. 12, no. 2, pp. 110-116, 2002. 
[8] L. Dalton, "Nonlinear optical polymeric materials: from chromophore design to commercial applications," Advances in Polymer Science, vol. 158, pp. 1-86, 2002.

[9] Y. Zhang, J. Martinez-Perdiguero, U. Baumeister et al., "Laterally azo-bridged $\mathrm{H}$-shaped ferroelectric dimesogens for secondorder nonlinear optics: ferroelectricity and second harmonic generation," Journal of the American Chemical Society, vol. 131, no. 51, pp. 18386-18392, 2009.

[10] D. Frattarelli, M. Schiavo, A. Facchetti, M. A. Ratner, and T. J. Marks, "Self-assembly from the gas-phase: dsign and implementation of small-molecule chromophore precursors with large nonlinear optical responses," Journal of the American Chemical Society, vol. 131, no. 35, pp. 12595-12612, 2009.

[11] G. J. Ashwell, "Langmuir-Blodgett films: molecular engineering of non-centrosymmetric structures for second-order nonlinear optical applications," Journal of Materials Chemistry, vol. 9, pp. 1991-2003, 1999.

[12] L. R. Dalton, S. J. Benight, L. E. Johnson et al., "Systematic nanoengineering of soft matter organic electro-optic materials," Chemistry of Materials, vol. 23, pp. 430-445, 2011.

[13] M. Jazbinsek, O. P. Kwon, C. Bosshard, and P. Gunter, "Organic nonlinear optical crystals and single-crystalline thin films," in Handbook of Organic Electronics and Photonics, S. H. Nalwa, Ed., American Scientific Publishers, 2008.

[14] D. Rezzonico, S. J. Kwon, H. Figi, O. P. Kwon, M. Jazbinsek, and P. Günter, "Photochemical stability of nonlinear optical chromophores in polymeric and crystalline materials," Journal of Chemical Physics, vol. 128, no. 12, Article ID 124713, 2008.

[15] H. Figi, L. Mutter, C. Hunziker, M. Jazbinšek, P. Günter, and B. J. Coe, "Extremely large nonresonant second-order nonlinear optical response in crystals of the stilbazolium salt DAPSH," Journal of the Optical Society of America B, vol. 25, no. 11, pp. 1786-1793, 2008.

[16] M. Jazbinsek, L. Mutter, and P. Günter, "Photonic applications with the organic nonlinear optical crystal DAST," IEEE Journal on Selected Topics in Quantum Electronics, vol. 14, no. 5, pp. 1298-1311, 2008.

[17] M. Tonouchi, “Cutting-edge terahertz technology," Nature Photonics, vol. 1, no. 2, pp. 97-105, 2007.

[18] K. Balasubramani, P. T. Muthiah, and D. E. Lynch, "2-Amino4,6-dimethylpyrimidine-4-hydroxybenzoic acid (1/1)," Acta Crystallographica Section E, vol. 62, no. 7, pp. o2907-o2909, 2006.

[19] K. A. Nandekar, J. R. Dontulwar, and W. B. Gurnule, "Thermoanalytical studies and kinetics of newly synthesized copolymer derived from P-hydroxybenzoic acid, and semicarbazide," Rasayan Journal of Chemistry, vol. 5, no. 3, pp. 261-268, 2012.

[20] R. A. Khatri, S. S. C. Chuang, Y. Soong, and M. Gray, “Thermal and chemical stability of regenerable solid amine sorbent for $\mathrm{CO}_{2}$ capture," Energy and Fuels, vol. 20, no. 4, pp. 1514-1520, 2006.

[21] C. N. R. Rao, Ultraviolet and Visible Spectroscopy, Butterworths, London, UK, 3rd edition, 1975.

[22] V. Krishnakumar and R. Nagalakshmi, "Crystal growth and vibrational spectroscopic studies of the semiorganic non-linear optical crystal: bisthiourea zinc chloride," Spectrochimica Acta A, vol. 61, no. 3, pp. 499-507, 2005.

[23] R. N. Rai, S. R. Mudunuri, R. S. B. Reddi, V. S. A. Kumar Satuluri, S. Ganeshmoorthy, and P. K. Gupta, "Crystal growth and nonlinear optical studies of $m$-dinitrobenzene doped urea," Journal of Crystal Growth, vol. 321, no. 1, pp. 72-77, 2011.
[24] V. Pandey, N. Mehta, S. K. Tripathi, and A. Kumar, "Optical band gap and optical constants in $\mathrm{Se}_{85} \mathrm{Te}_{15-x} \mathrm{~Pb}_{x}$ thin films," Chalcogenide Letters, vol. 2, no. 5, pp. 39-44, 2005.

[25] M. Dongol, "Optical absorption and structural properties of as-deposited and thermally annealed As-Te-Ga thin films," Egyptian Journal of Solids, vol. 25, no. 1, pp. 33-47, 2002.

[26] R. E. Denton, R. D. Campbell, and S. G. Tomlin, "The determination of the optical constants of thin films from measurements of reflectance and transmittance at normal incidence," Journal of Physics D, vol. 5, pp. 852-863, 1972.

[27] A. Ashour, N. El-Kadry, and S. A. Mahmoud, "On the electrical and optical properties of CdS films thermally deposited by a modified source," Thin Solid Films, vol. 269, no. 1-2, pp. 117-122, 1995.

[28] G. C. Bhar, A. K. Chaudhary, and P. Kumbhakar, "Study of laser induced damage threshold and effect of inclusions in some nonlinear crystals," Applied Surface Science, vol. 161, no. 1, pp. 155$162,2000$.

[29] H. L. Bhat, "Growth and characterization of some novel crystals for nonlinear optical applications," Bulletin of Materials Science, vol. 17, no. 7, pp. 1233-1249, 1994.

[30] K. Nagarajan and C. K. Shasidharan Nair, "The use of fractional orders in the determination of birefringence of highly dispersive materials by the channelled spectrum method," Optics Communications, vol. 275, pp. 348-353, 2007.

[31] N. Vijayan, K. Nagarajan, A. M. Z. Slawin, C. K. S. Nair, and G. Bhagavannarayana, "Growth of benzimidazole single crystal by sankaranarayanan-ramasamy method and its characterization by high-resolution X-ray diffraction, thermogravimetric/ differential thermal analysis, and birefringence studies," Crystal Growth and Design, vol. 7, no. 2, pp. 445-448, 2007.

[32] N. Vijayan, G. Bhagavannarayana, K. Nagarajan, and V. Upadhyaya, "Synthesis and growth of nearly perfect single crystal of 1-histidine bromide (LHB) and its structural, optical and electrical characterizations," Materials Chemistry and Physics, vol. 115, no. 2-3, pp. 656-659, 2009.

[33] S. K. Kurtz and T. T. Perry, "A powder technique for the evaluation of nonlinear optical materials," Journal of Applied Physics, vol. 39, no. 8, pp. 3798-3813, 1968. 

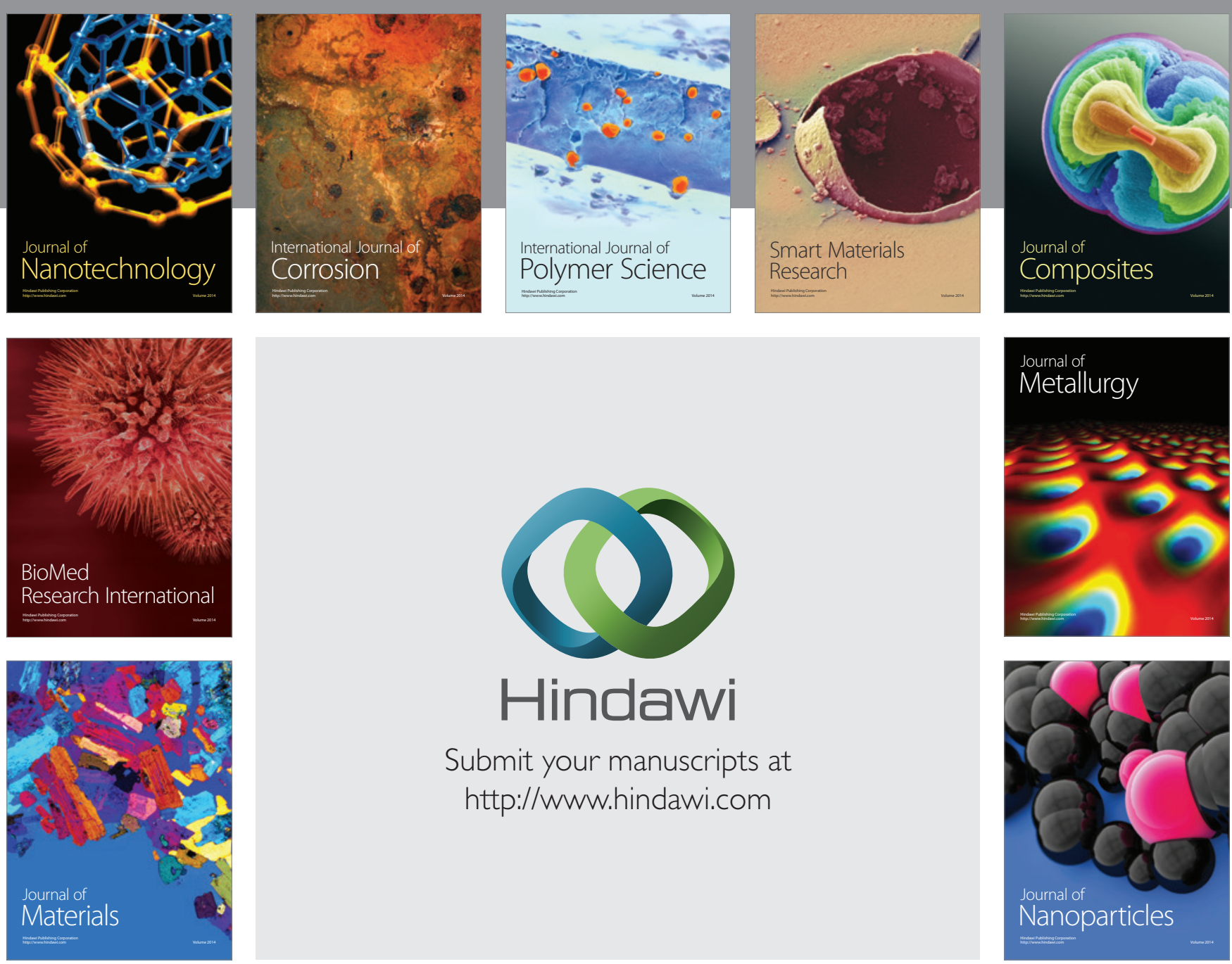

Submit your manuscripts at http://www.hindawi.com
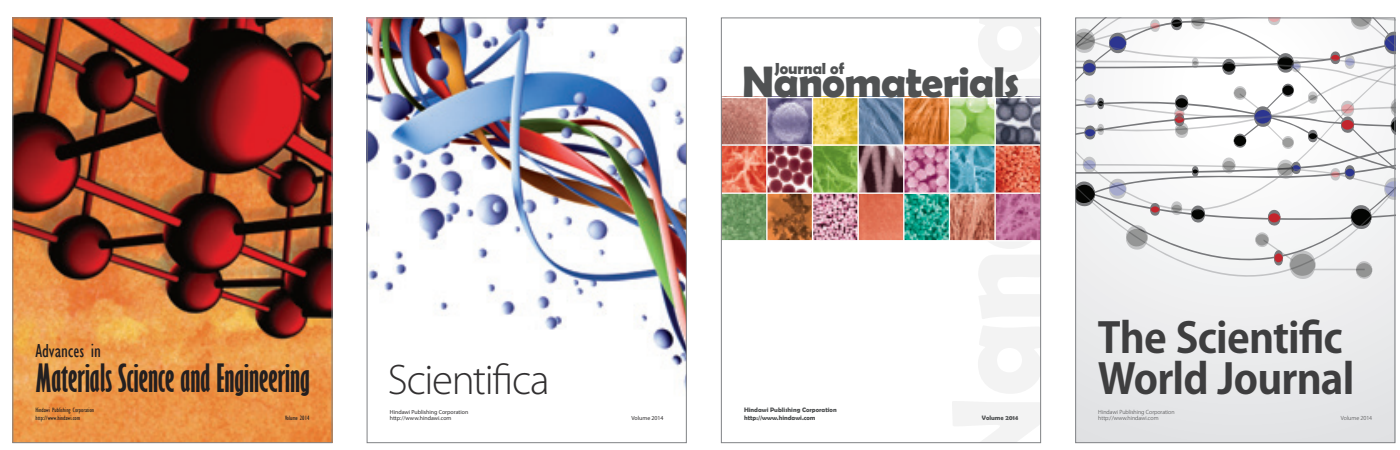

\section{The Scientific World Journal}
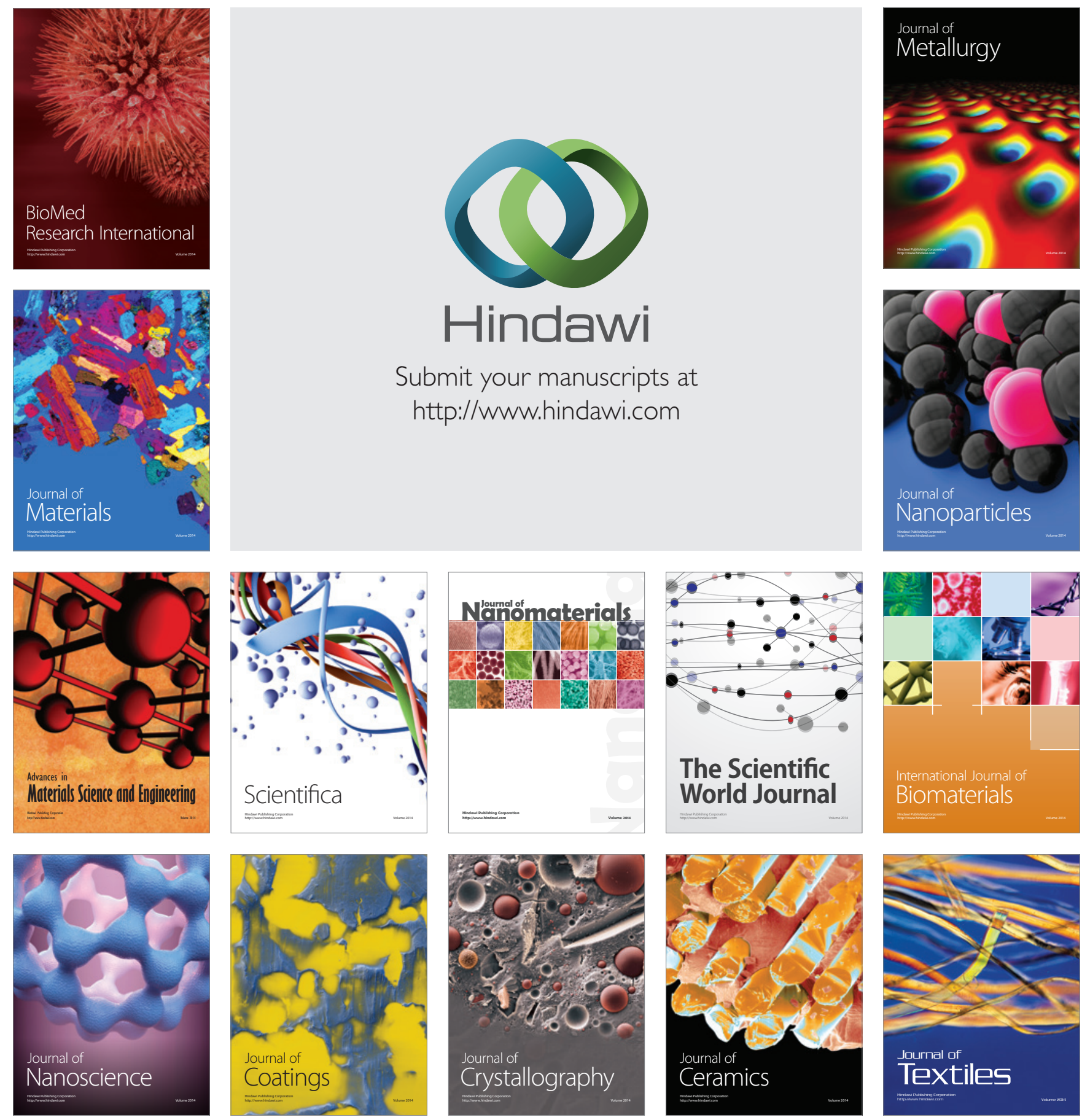